\title{
ESTIMACIÓN DE RIESGO DE DESASTRE EN LAS QUEBRADAS DE SANTA CECILIAY PILACUCHO DEL BARRIO SANTA ANA, AYACUCHO, 2014
}

\author{
FREDDY LEÓN NINA ${ }^{1}$ \\ Universidad NACIONAL SAN CRISTÓbal DE HUAMANGA \\ leondeosqonta@outlook.com
}

\section{RESUMEN}

El estudio sistemático de la vulnerabilidad de las quebradas de Santa Cecilia, La Unión y Pilacucho del Barrio de Santa Ana, tiene como objetivo generar conocimiento sobre el riesgo-desastre en sus diferentes ámbitos; estimación, frecuencia, cálculo y análisis de riesgo. Tomando en cuenta la amenaza natural existente como geológicas, hidrometeorológicas y ambientales ante los escenarios del Cambio Climático (CC); la marginalización de las familias rurales en su mayoría han llevado a establecerse en terrenos deleznables cada vez menos seguros físicamente y los niveles de pobreza han aumentado continuamente con consecuencias nefastas porque aumentan el riesgo social y la vulnerabilidad a los desastres. Aplicamos toda una metodología de Parámetros y Niveles de Vulnerabilidad recomendadas por el Centro Nacional de Estimación, Reducción y Prevención del Riesgo Desastre (CENEPRED) y, como resultado se obtuvo; la Quebrada de Pilacucho, es una zona crítica expuestos a peligros recurrentes de mayor incidencia como la inundación y la contaminación ambiental, que representan RIESGOS MUY ALTOS. En cambio, el índice de prevalencia de vulnerabilidad es de 80\%, considerada VULNERABILIDAD MUY ALTA, lo que significa las poblaciones tienen deficientes respuestas para enfrentar los efectos negativos de los desastres naturales y ambientales.

Palabras claves: Riesgo desastres amenazas naturales y tecnológicos análisis de vulnerabilidad

\section{Abstract}

The systematic study of the vulnerability of the streams of Santa Cecilia, La Union and Pilacucho of the Santa Ana, aims to generate knowledge on disaster-risk in different areas; estimation, frequency, calculation and risk analysis. Considering the existing natural hazard and geological, hydrometeorological and environmental scenarios to climate change (CC); marginalization of rural families have mostly been established in despicable land increasingly less secure physically and poverty levels have

1 Profesor Auxiliar del Área de Geografía de la Facultad de Ciencias Sociales, de la Universidad Nacional de San Cristóbal de Huamanga, Email leondeosqonta@outlook.com. 
increased steadily with dire social consequences because they increase the risk and vulnerability to disasters. We use a whole methodology of parameters and levels recommended by the National Center for Estimation, Risk Reduction and Disaster Prevention (CENEPRED) and as a result was obtained Vulnerability; Pilacucho Quebrada is a critical area exposed to higher incidence of recurrent hazards such as flooding and pollution, which represent very high risks. Instead, the vulnerability prevalence rate is $80 \%$, considered highly vulnerable, which means populations have poor responses to address the negative effects of natural and environmental disasters. It is recommended to develop a Comprehensive Management Plan of the Downs.

KeYwoRds: Natural hazards disaster risk and vulnerability analysis technology

\section{INTRODUCCIÓN}

El tema de prevención de desastres como fenómeno de origen natural y ambiental, tiene un interés en la agenda regional como prioridad para el desarrollo económico y social. La investigación de Estimación de Riesgo en las Quebradas de Santa Cecilia, La Unión y Pilacucho en el Barrio de Santa Ana de la ciudad de Ayacucho, nos permite analizar las viviendas y poblaciones asentadas en las quebradas frente a los eventos de peligros y vulnerabilidades; con el propósito de estimar el nivel de riesgo existente en la zona del "alud 2009", y las zonas adyacentes que van colmatando las fajas marginales de las quebradas en las que están expuestas las edificaciones existentes y las poblaciones. Los objetivos son:

- Hacer línea de base de causas y efectos de los peligros, vulnerabilidad y riesgo de los desastres naturales y antrópicos en las quebradas de Santa Cecilia, La Unión y Pilacucho del distrito de Ayacucho.

- Generar conocimiento sobre el riesgo-desastre en sus diferentes ámbitos; estimación, frecuencia, cálculo y análisis de riesgo.

Las metodologías y herramientas estipuladas en el CENEPRED (Centro Nacional de Estimación, Prevención y Reducción del Riesgo de Desastre), permiten analizar las vulnerabilidades ambientales, económicas, sociales y de infraestructura planteadas en los objetivos. En el procesamiento y análisis de los datos de trabajo de campo, ha contribuido a la interpretación de riesgo desastres, para determinar el índice de vulnerabilidad prevalente (IVP) causados por las amenazas sísmicas, hidrometeorológicos y geológicos, que se traducen en las vulnerabilidades de los asentamientos humanos, para tener una información del grado de inestabilidad de las quebradas consideradas de alto riesgo, de tal manera, se deben mitigar con planes de contingencia y adaptarnos al cambio climático.

Martínez (2009) ha recopilado vocablos de la época clásica, desde los orígenes de los estudios sobre fenómenos naturales peligrosos se utilizaron dos palabras: una, desastre, que procede del prefijo latino des (falta de, malo) y de la palabra griega astron o astren (estrella); en términos literales, desastre sería "mala estrella", que implica infortunios o calamidades o, en todo caso, acontecimientos que se imponen inexorablemente a las acciones y voluntades humanas. La otra palabra muy utilizada en la antigüedad es katástrophé, que significa ruina o desgracia, y de strepo, que significa volverse, y se refiere a un suceso fatídico en que hay gran destrucción y que altera el orden regular de las cosas.

De acuerdo con Narváez et al., (2009) históricamente, la definición de "riesgo de desastre" ha tomado dos rumbos: En primera instancia están las definiciones que se derivan de las ciencias de la Tierra y que tienden a definir el riesgo como "la probabilidad de la ocurrencia de un evento físico dañi- 
no". Esta definición pone énfasis en la amenaza o el evento físico detonador del desastre. En segunda instancia, están las definiciones de riesgo desastre que rescatan lo social y lo económico y tienden a plasmarse en definiciones del siguiente tipo: "el riesgo desastre comprenden la probabilidad de daños y pérdidas futuras asociadas con la ocurrencia de un evento físico dañino". Ósea, el énfasis se pone en los impactos probables y no en la probabilidad de ocurrencia del evento físico como tal.

Como sabemos, el riesgo es diferenciado según el contexto y la escala geográfica o espacial en el que se analice. No todas las comunidades y territorios presentan iguales condiciones de amenazas y vulnerabilidad, ni tampoco de materializarse el riesgo, el impacto ocasionado será igual para todos los afectados. Un mismo evento puede ocasionar diferentes niveles de daño o pérdidas para distintos segmentos de la población o territorios según las condiciones de vulnerabilidad en que se encuentren; de ahí, es que surgen las necesidades de dimensionar los problemas, las soluciones y las estrategias de gestión de riesgo - desastre.

Para Ocharam (2008) el objetivo fundamental en torno a la reducción de riesgo es conseguir que la población susceptible de padecer los efectos de una amenaza no la sufra transformada en un desastre. La vulnerabilidad tiene diferentes niveles y factores (individual, físico, social, económico y medioambiental) y que junto con la exposición (entendida como parte de la vulnerabilidad o independiente de ella) son fuerzas que se hallan en el numerador y, por tanto, que deben ser reducidas si se quiere un valor numérico final menor. En cambio, la capacidad, que también puede ser entendida en sus diferentes variables, como las estrategias de afrontamiento o la resiliencia, es un valor que se debe ser aumentado. Todo lo anterior, en combinación con la amenaza, que también tiene que ser reducida en la medida de lo posible, termina por componer el riesgo.

Los peligros de origen geológico y geológico-climático de mayor incidencia en la Ciudad de Ayacucho, son amplificación local de ondas sísmicas, deslizamientos, socavamientos y cárcavas, desprendimientos de rocas y derrumbes de suelos, procesos que se ven favorecidos por una alta pendiente del terreno, baja o pobre resistencia de los materiales y el agua de lluvia en su acción de erosión, transporte y deposición (Matos 2003).

Así, como evitar la generación de nuevos riesgos, preparación y atención ante situación de desastres mediante los componentes, procesos e instrumentos de la gestión de riesgo de desastre. Reducir la fragilidad a nivel físico (caso de la resistencia de edificaciones como las viviendas) y funcional-organizativo (caso de la supervivencia de los servicios básicos como salud, educación y saneamiento) que es importante para conservar un nivel mínimo de bienestar.

\section{Materiales y Métodos}

Para la Estimación de Gestión de Riesgo-Desastres de la Quebrada de Santa Cecilia, Unión Pilacucho del Barrio de Santa Ana, se han desarrollado las siguientes metodologías y actividades:

Se ha utilizado el método multicriterio (proceso de análisis jerárquico) para la ponderación de los parámetros de evaluación del fenómeno de origen natural y de la vulnerabilidad, mostrando la importancia de cada parámetro en el cálculo de riesgo, facilitando la estratificación de los niveles de riesgos. Este método permite incorporar información cuantitativa (mediciones de campo) y cualitativa (nivel de incorporación de los instrumentos de gestión del riesgo, niveles de organización social, etc.).

Para la evaluación cualitativa de riesgos implica el conocimiento de los peligros, de los elementos expuestos y de sus vulnerabilidades, basado en la experiencia y observaciones de campo debido a la 
inexistencia de información (registros históricos, estadísticos, estudio técnicos, etc.) del fenómeno de origen natural sobre el área geográfica de estudio (CENEPRED 2013).

Para el levantamiento de la información de campo y registro fotográfico estamos utilizando las siguientes técnicas y materiales:

- Carta Nacional de IGM: Escalas 1:100,000 y 1:25,000

- Carta Geológica Nacional: Escala: 1: 100,000 (Hoja 26-ñ Ayacucho).

- Matriz de Zonificación de Riesgos.

- GPS GARMIN MONTANA 650: (cámara 5.0 megapíxeles, memoria interna 3GB, compás electrónico, brújula, altímetro barométrico).

- Cámara Fotográfica DCM-FH25 8X16 MEGA PIXELS.

- Recopilación de Información existente de varias instituciones y bibliotecas virtuales.

- Investigación de Campo, han permitido colectar "in situ" toda la información de geología, geomorfología, hidrometeorología y sociodemográfica del área de interés para confirmar y completar la información seleccionada.

- Labores de Gabinete, se han desarrollado con los resultados obtenidos anteriores y su objetivo es la identificación de peligros y el análisis de vulnerabilidades en la población dada.

La vulnerabilidad total, se calcula en base al promedio obtenido por cada tipo de vulnerabilidad a través de la siguiente formula:

$$
V T=V F+V A e+V E+V S+V e d+V P I+V C I+V C T
$$

\section{8}

En donde:

$\begin{array}{lll}\text { VT } & = & \text { Vulnerabilidad Total } \\ \text { VF } & = & \text { Vulnerabilidad Física } \\ \text { VAE } & = & \text { Vulnerabilidad Ambiental }- \text { Ecológica } \\ \text { VE } & = & \text { Vulnerabilidad Económica } \\ \text { VS } & \text { Vulnerabilidad Social } \\ \text { Ved } & \text { Vulnerabilidad Educativa } \\ \text { VPI } & \quad \text { Vulnerabilidad Política Institucional } \\ \text { VCI } & \quad \text { Vulnerabilidad Cultural - Ideológica } \\ \text { VCT } & \quad \text { Vulnerabilidad Científica }- \text { Tecnológica. }\end{array}$


Parámetros y Niveles de Vulnerabilidad

\begin{tabular}{|l|l|l|}
\hline $0-15 \%$ & Bajo & Zonas de vulnerabilidad Baja \\
\hline $15-30 \%$ & Medio & Zonas de vulnerabilidad Media \\
\hline $30-60 \%$ & Alto & Zonas de vulnerabilidad Alto \\
\hline $60-100 \%$ & Muy Alto & Zonas de vulnerabilidad Muy Alto \\
\hline
\end{tabular}

Fuente: CISMID, PREDES, 2006 (http://www.observatoriourbano.org.pe).

\section{Resultados}

\section{Ubicación geográfica}

La zona en estudio se encuentra geográficamente entre las Coordenadas UTM:

- Norte : 8’544,596.620 - 8'544,390.970 m.

- Este : 583,647.430 - 582,929.010 m.

- $\quad$ Altitud : 2,705.690 - 2,758.110 m.s.n.m.

- Área evaluada:

El tramo evaluada es de $3.0 \mathrm{Km}$ lineales de la mini cuenca de Pilacucho, desde de la divisoria acuática hasta el colector del río Alameda, comienza entre las Quebrada de Huascahura y la Q. Pilacucho, que nace del Co de Cabrapata a 3,292 msnm y Q. Unión - Santa Cecilia nace de las laderas empinadas de Santa Ana; los interfluvios, son espacios comprendidos que forma una topografía triangular entre dos cuenca vecinas y drenan directamente al río receptor del río Seco y río Alameda.

El Barrio de Santa Ana, presenta una superficie de 14.05 ha que representa el $0.76 \%$ de la superficie ocupada por la ciudad de Ayacucho, concentra una población de 1,124 habitantes que ocupan en promedio 225 viviendas, con una densidad promedio de $80 \mathrm{hab} / \mathrm{ha}$. Compromete a las zonas ubicadas sobre ambas márgenes de los ramales de la quebrada Pilacucho (Fig. 1.).

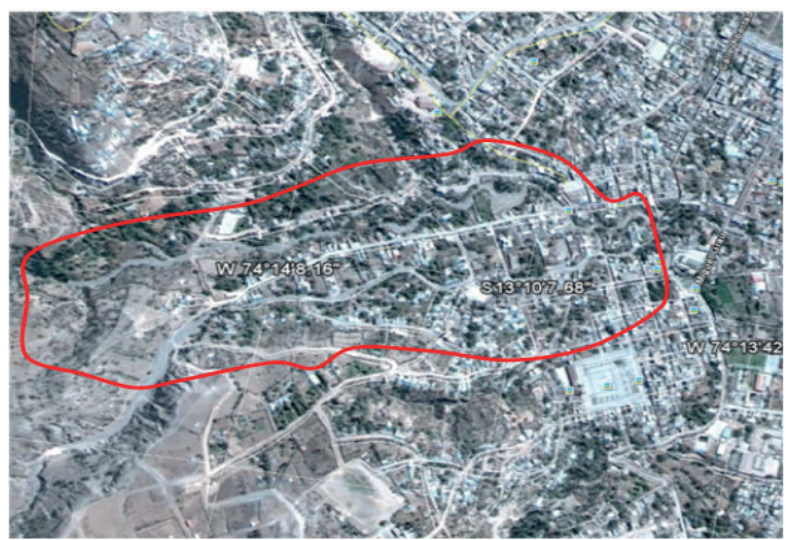

Fig. 1. Vista satelital y panorámica de la unidad de análisis: Quebrada Unión Santa Cecilia-Santa Ana y Pilacucho, Ciudad de Ayacucho. 
Ambas quebradas están expuestas a las amenazas de los fenómenos de origen geológico2 y hidrometeorológico, relacionados a la acción pluvial, presentándose inundaciones por las crecidas repentinas en los periodos lluviosos, la erosión intensa van formando los surcos y cárcavas en los alrededores de la quebrada, que pone en riesgo las edificaciones construidas cercanas a ésta, ubicadas particularmente en el Sector denominado Pueblo Joven La Unión, el cauce sirve como calles y pasajes de entrada y salida de las casas vecinales.

Geológicamente la Ciudad de Ayacucho y su entorno está emplazada sobre depósitos de suelos volcánicos sedimentarios cuyas edades oscilan entre Terciario Superior y Cuaternario Reciente. La cuenca de la ciudad de Ayacucho, es resultado de la formación geológica Cretácico-Terciario-Volcánico. La quebrada del interfluvio Unión Santa Cecilia y Pilacucho, está constituido de masas solidificadas de lavas volcánicas que han formado las rocas eruptivas de tufos y tobas volcánicas3.

La topografía del Cerro Cabrapata es empinada de índice de masividad muy montañoso, en el cual nos señala que ésta mini cuenca de Pilacucho, es muy peligroso por su configuración litológica. En estaciones lluviosas, la carga erosiva inunda los lechos y cauces de las quebradas, la zona presentan valles angostos, huaycos y cárcavas que atraviesan gargantas abruptas y terminan en fondos estrechos con ocupaciones de viviendas, con cotas que varían desde los 2,760 msnm hasta los 3,200 msnm (Figura 1).

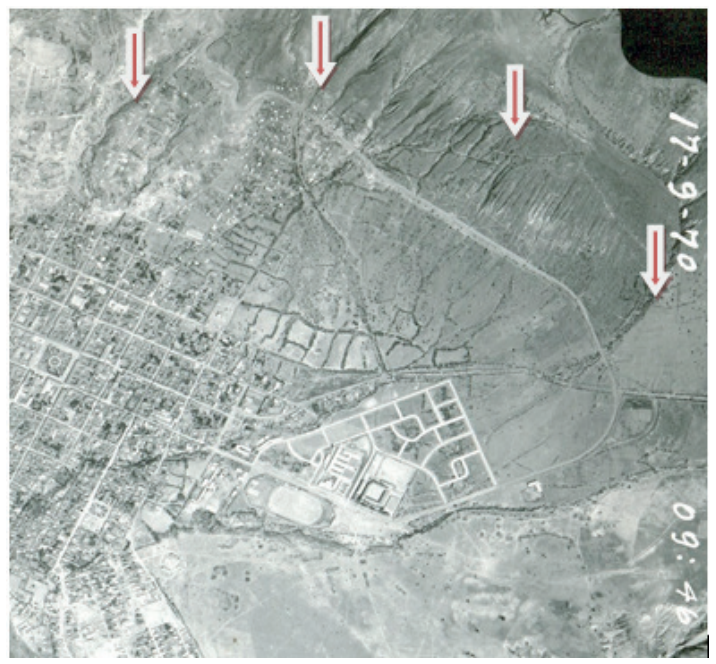

Fig. 1. Fotografía Aérea de la franja del co La Picota y parte de Co Cabrapata. Servicio Aerofotográfico Nacional (1970).

2 El Perú es un territorio vulnerable a los huaycos -desplazamiento violento de una gran masa de lloclla con lodo y rocas que se movilizan a gran velocidad por quebradas y valles- debido a la topografía montañosa altitudinal. Un huayco, se origina con precipitaciones torrenciales asociadas a todo tipo de movimiento de masas y efectos sísmicos. Arrasa todo lo que encuentra en su trayecto que genera los desastres. El caso del alud del Co La Picota de la Ciudad de Ayacucho en 2009, las precipitaciones intensas de $52.8 \mathrm{~mm}$, en 2 horas fueron lluvias intensas para el desplazamiento de masas y huayco trajo la avalancha de lodo, rocas y materiales sedimentarios que sepultó a 10 personas fallecidas y la destrucción de la infraestructura y servicios, la ladera es considerada de Muy Alta Vulnerabilidad. 3 Las tobas volcánicas de Formación Ayacucho, se caracterizan por tener aspecto masivo, color rosado, composición riolítica a dacítica, textura porfirítica y buena compactación, ocupan los Cํ de La Picota y Cabrapata. 
Según el Servicio Aerofotográfico Nacional (1970). La Ladera del Co la Picota, Huascahura y Cº Cabrapata; es una montaña rocosa de formación volcánica y sedimentaria, con una serie de redes de drenaje naturales areno limosos bien graduada de origen sedimentario antiguo (conglomerado pleistoceno); sin embargo, la topografía fue discontinua por el material rocoso separados, por planos de debilidad como estratificación y exfoliación claramente se diferencian los colectores naturales en la margen izquierda del río Alameda. La microcuenca recepciona la carga pluvial y los materiales sedimentarios hacia el fondo del río Alameda. Mediante la observación e interpretación de fotografías aéreas y fotografías en el terreno; se considera una amenaza constante en los riesgos y sus efectos adversos en la población de la ciudad de Ayacucho y prevalecen altos niveles de vulnerabilidad.

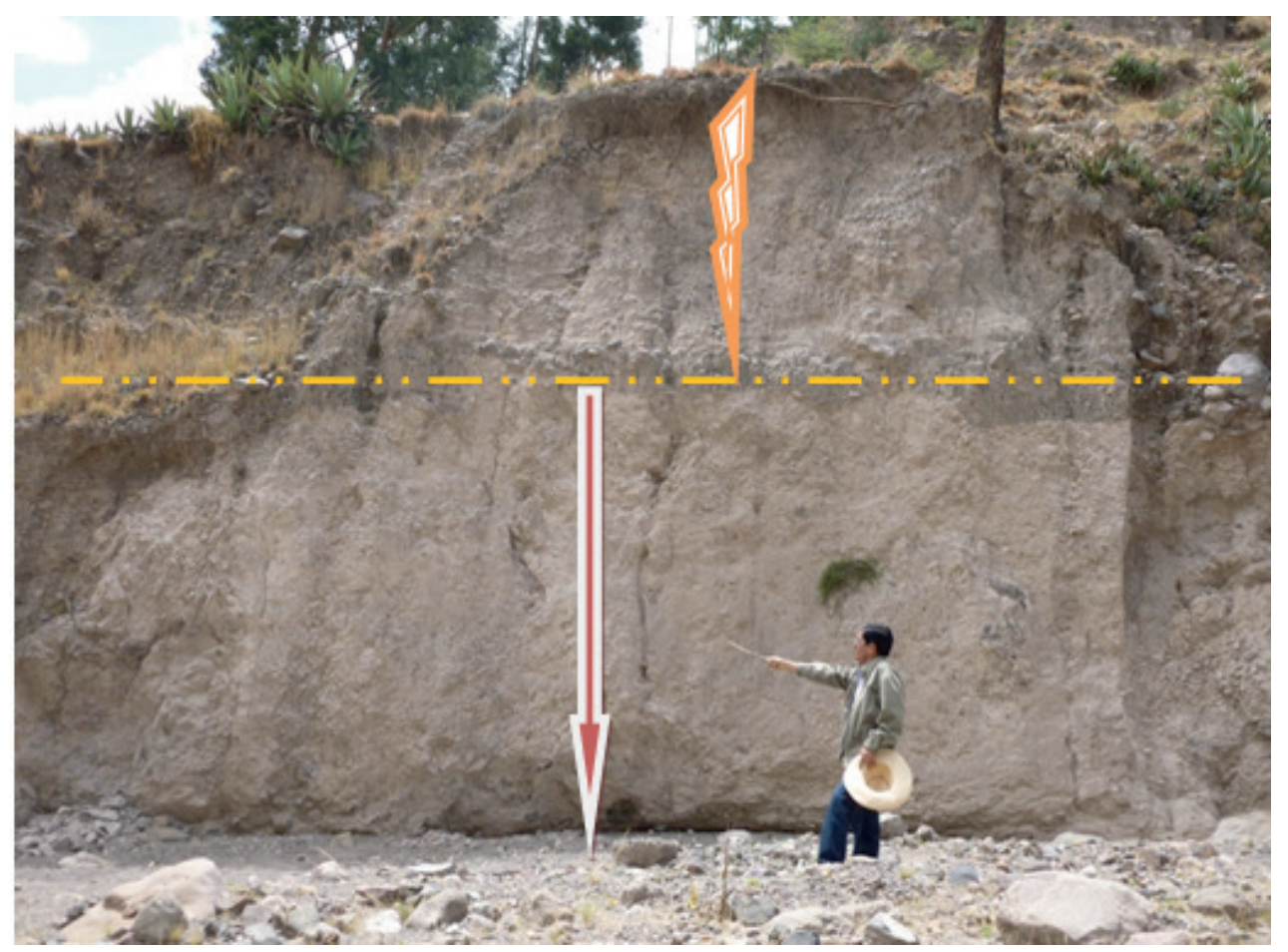

Fig. 2 Estratos geológicos de tufo volcánico y materiales sedimentarios aluviales en la cuenca intermedia de la $Q$. Unión Santa Cecilia.

El tipo climático para la cuenca de la Ciudad de Ayacucho corresponde una sensación térmica de carácter "templado" con una t ${ }^{\circ}$ promedio anual de $17.3^{\circ} \mathrm{C}$ propia de una zona semiárida en quebradas secas y laderas empinadas de Co La Picota, Huascahura y Cabrapata. Las precipitaciones pluviales en la cuenca de la Ciudad de Ayacucho, fue de $680.10 \mathrm{~mm} /$ año. Esto significa, una amplitud pluviométrica muy variada, las épocas de mayor precipitación corresponde al verano lluvioso de diciembre a marzo; mientras, la sequedad y ausencia de lluvias corresponde al invierno seco de mayo a julio; en consecuencia, las precipitaciones de la ciudad de Ayacucho es marcadamente diferenciada en su régimen climático; las lluvias ocurren en verano lluvioso de diciembre a marzo, mientras tenemos una estación de invierno seco de mayo a agosto para estas regiones. 
Los estudios realizados por León (2009) dice los siguiente; la ciudad de Ayacucho, flagelada por el desastre de un "alud" originado por lluvias focalizadas en la montaña del Co La Picota, se ha registrado precipitaciones de fuerte intensidad a lo largo de la región andina. SENAMHI (2009) aclaró, lo ocurrido de la lluvia en el Cerro La Picota de la Ciudad de Ayacucho, no tuvo relación alguna con el Fenómeno de "El Niño", sino con la variabilidad climática, se formó un núcleo (masa nubosa) que ocasionó una lluvia de nivel fuerte. Según los datos registrados en la Estación Killa (Piscotambo, Ayacucho), las precipitaciones intensas del 16 de diciembre del 2009 fue de $52.8 \mathrm{~mm}$, en un tiempo record de 2 horas, se inició a las $5.30 \mathrm{pm}$ hasta $7.30 \mathrm{pm}$, que fueron suficientes para crear un huayco moderno, que trajo la avalancha de materiales gravosos y rocas volcánicas que colmataron las quebradas y los colectores naturales de la ladera. Paralelamente, las precipitaciones acumulados en la Estación del Pampa del Arco fue de $21.3 \mathrm{~mm}$ (Estación Huamanga: 2009), pero la tragedia no solo fue por ese evento de desastre hidrometeorológico y geológico; sino aquí intervienen la mismas amenazas socio-naturales (desaparición de huaycos y cárcavas) que van ocupando los cauces y quebradas con relleno y contaminación ambiental, producto de la pobreza urbana que ocupan las laderas de terrenos deleznables, que no presta seguridad física en un asentamiento humano y están expuestos a peligros cobrando vidas humanas y pérdida económicas que ha interrumpido el desarrollo de la población (ver Figura 3).

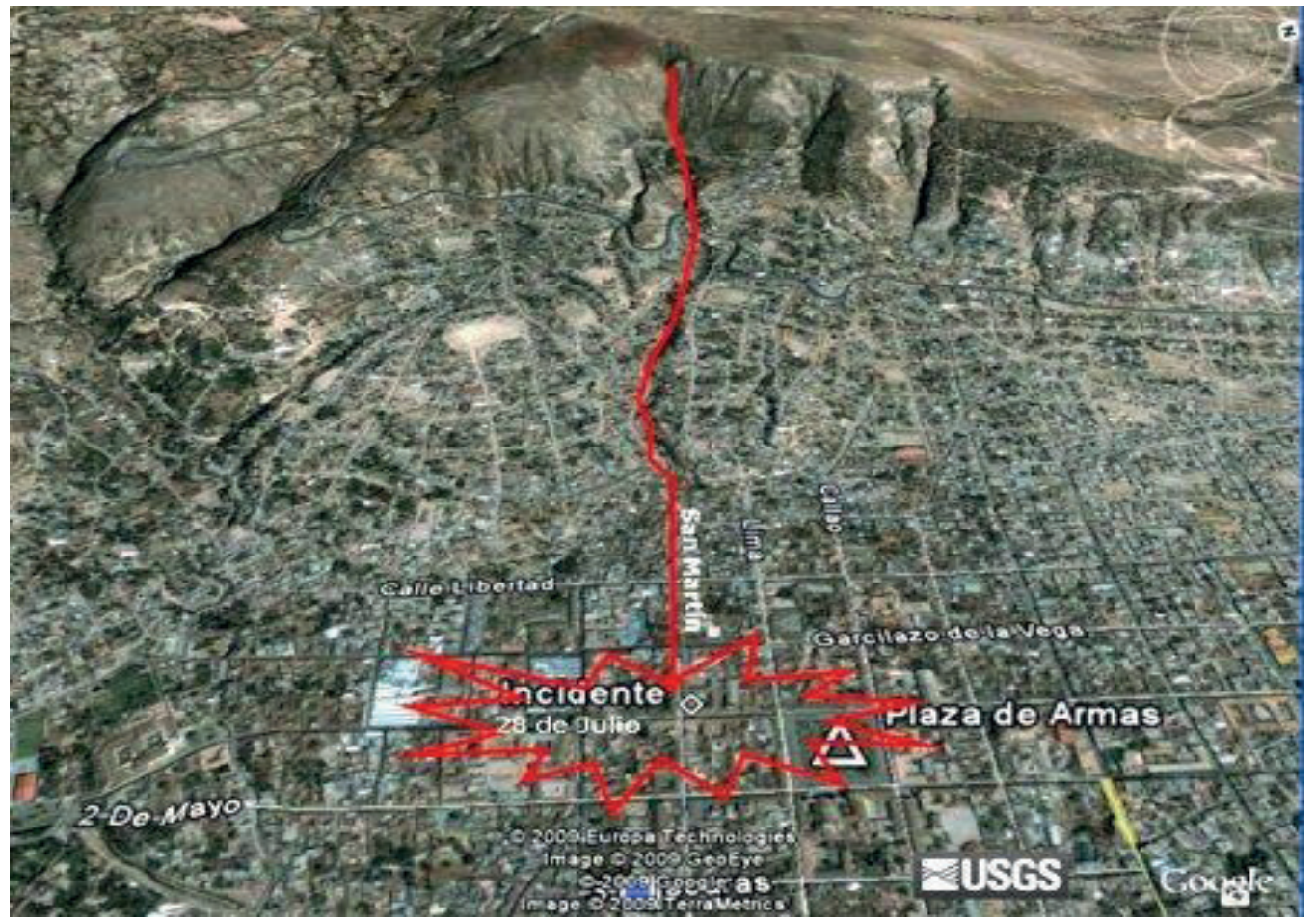

Fig. 3: Zona de desastre en la Ciudad de Ayacucho / Fuente: Agencia Andina 2009.

En base a la figura de Agencia Andina (2009), el huayco está ubicado a una altitud de 3,000 msnm, exactamente en la Capilla del Señor de San Cristóbal de Verde Verde, y la Cruz Blanco de la Pacificación. El recorrido del alud es de $1.8 \mathrm{Km}$ en línea recta con pendiente topográfico de $80^{\circ}$ hasta llegar al 
cruce de Jr. 28 de Julio y San Martín. El primer centro urbano afectado es el AA. HH. de Pueblo Libre, ubicado en la ladera de $\mathrm{C}^{\circ}$ La Picota, han sido arrasados sus calles, viviendas, sistema de alcantarillado de agua potable, desagüe y la red eléctrica, el desastre resultado del flujo de descarga pluvial, en los cuales los granos mineralógicos o elementos individuales son dispersados a través de un medio líquido de transporte denominado "flujo de detritos"; la remoción en mención, es caracterizado como "huaycos", se definen como un flujo local y repentino o torrente de volumen relativamente grande y de corta duración, que desborda las cauces de las quebradas, transportando una enorme carga de barro y fragmentos angulosos de rocas, generalmente vinculados a lluvias intensas muy esporádicas de corta duración ligados al cambio climático.

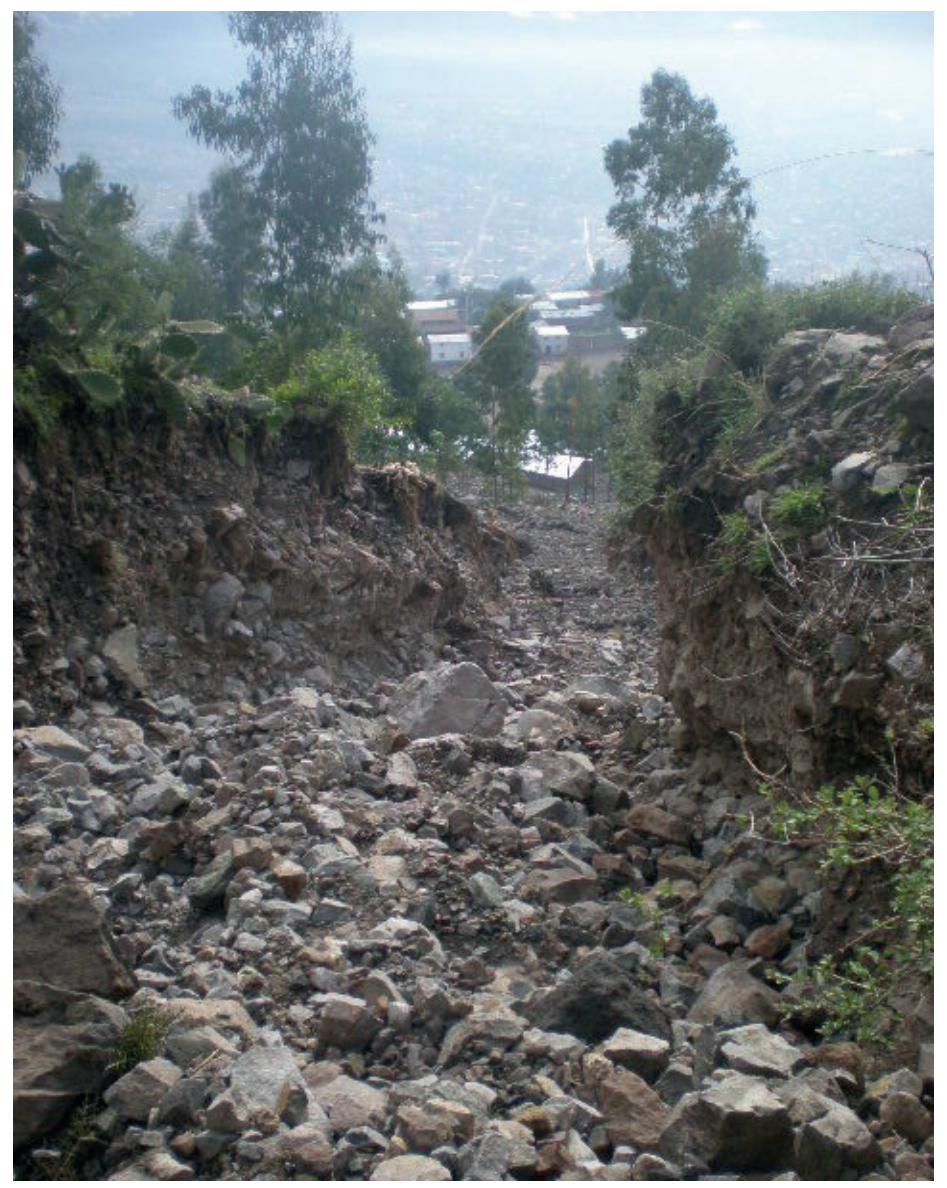

Fig. 4: Cauce nuevo y lecho gravoso del alud 16/12/2009. 


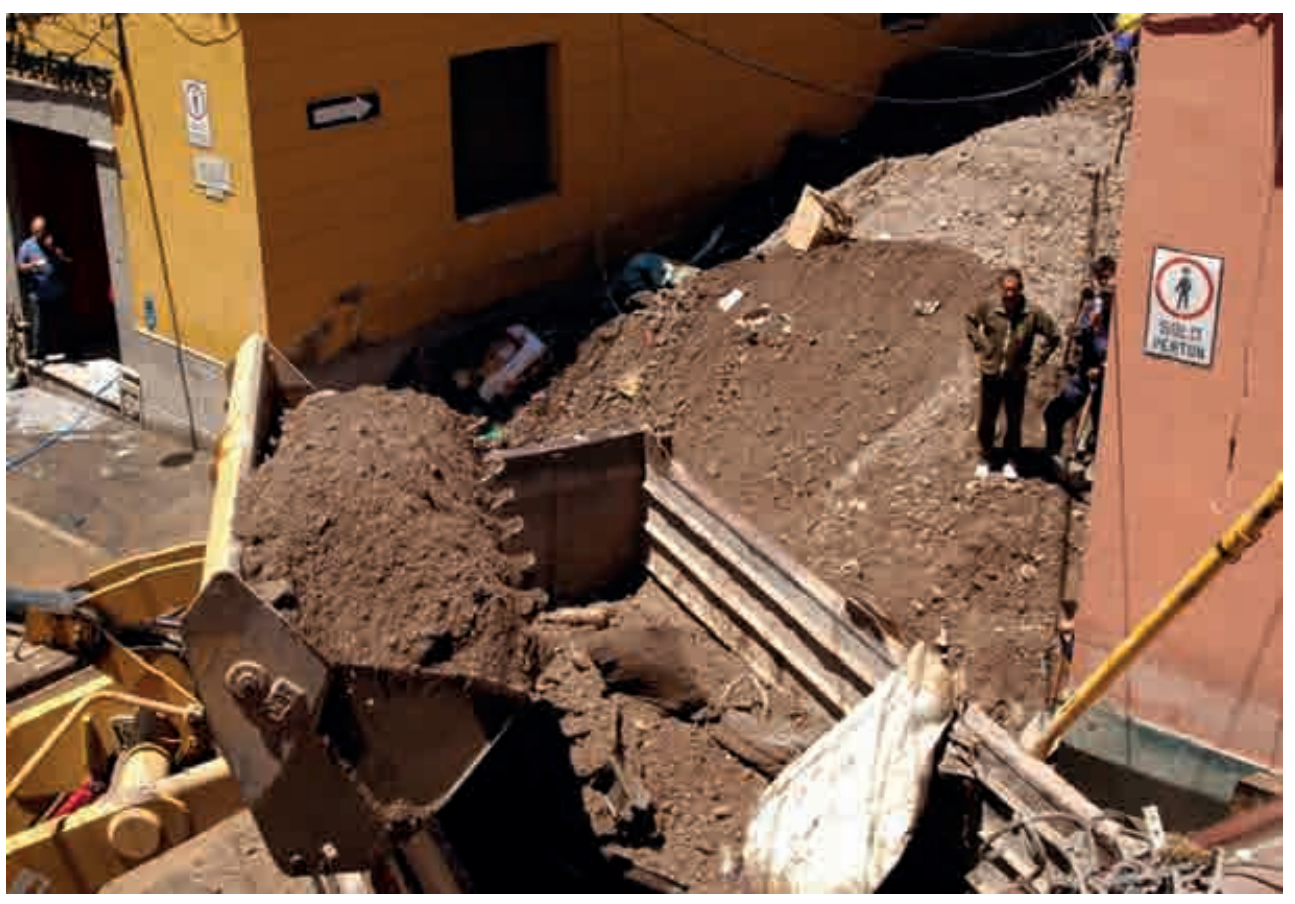

Fig. 5: Acumulación de material acarreado y depositado entre el Jr. 28 de Julio y San Martín: 17/12/ 2009.

La red de drenajes del río Alameda tiene varios afluentes a lo largo de su recorrido, entre las cuales destacan por su magnitud, la quebrada de Santa Cecilia y Pilacucho que confluyen en el puente Sotocc Chaca, que tienen su origen en los cerros de "Cabrapata", "Huascahura" y el abra Pilacucho; el cual resulta ser un agente erosivo importante que activan los materiales coluvio-aluvial. Las vertientes se encuentra atravesada por quebradas y cumplen la función de colectores naturales de las aguas pluviales principalmente los meses de diciembre a marzo, los mismos que desembocan al río Alameda que atraviesa la ciudad de Sur a Norte. Precisamente por las quebradas de Santa Cecilia y Pilacucho, discurren gran volumen de agua cuando se producen los eventos de las lluvias torrenciales en la cuenca alta y a lo largo de ellas, se calcula que en total por ambas quebradas discurren unos $70 \mathrm{~m} 3$ que finalmente drena al rio Alameda (Ver Fig. 6). 


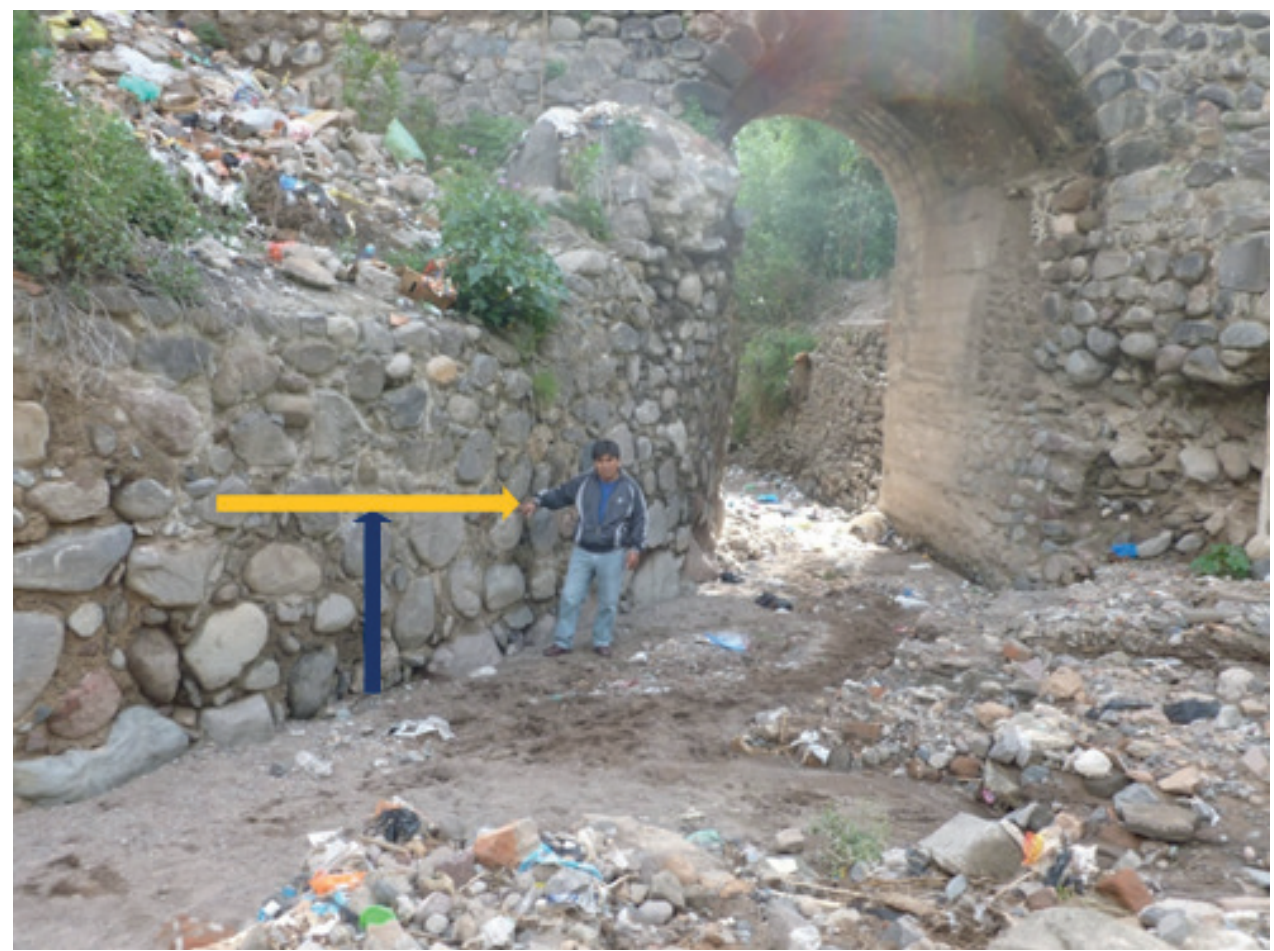

Fig. 6 Evidencias del caudal de $80 \mathrm{Cm}$ de altura aproximadamente que alcanza en el Puente de Sotocchaca en periodo de crecida y avenida de huaycos.

Entre las asociaciones naturales que corresponde altitudinalmente es la zonas de vida: Estepa Espinoso Montano Bajo Subtropical (ee - MBS), se extiende por toda la subcuenca de Q. Pilacucho, que van desde 2,780 hasta 3,100 msnm zonas bien definidas orográficamente y climáticamente. La biotemperatura media anual es de $16.2{ }^{\circ} \mathrm{C}$ y con una precipitación de $553.4 \mathrm{~mm} /$ año (promedio de 47 años de registro meteorológico de Pampa de Arco, UNSCH). La provincia de humedad es de semiárido, esto se entiende que la evapotranspiración potencial es de 2 a 4 veces de precipitación, en efecto, existe la carencia de humedad y las asociaciones de los vegetales se adaptan al xerofitismo, como la "tuna de altura" (Opuntia ficus - indica), el "molle" (Schinus molle), "maguey" (Agave americana), "cactáceas columnares" (Trichoreus peruvianus) y la "chamana" (Dodonaea viscosa) arbusto perennifolios y resistente a la sequía estacional (Fig. 7). 


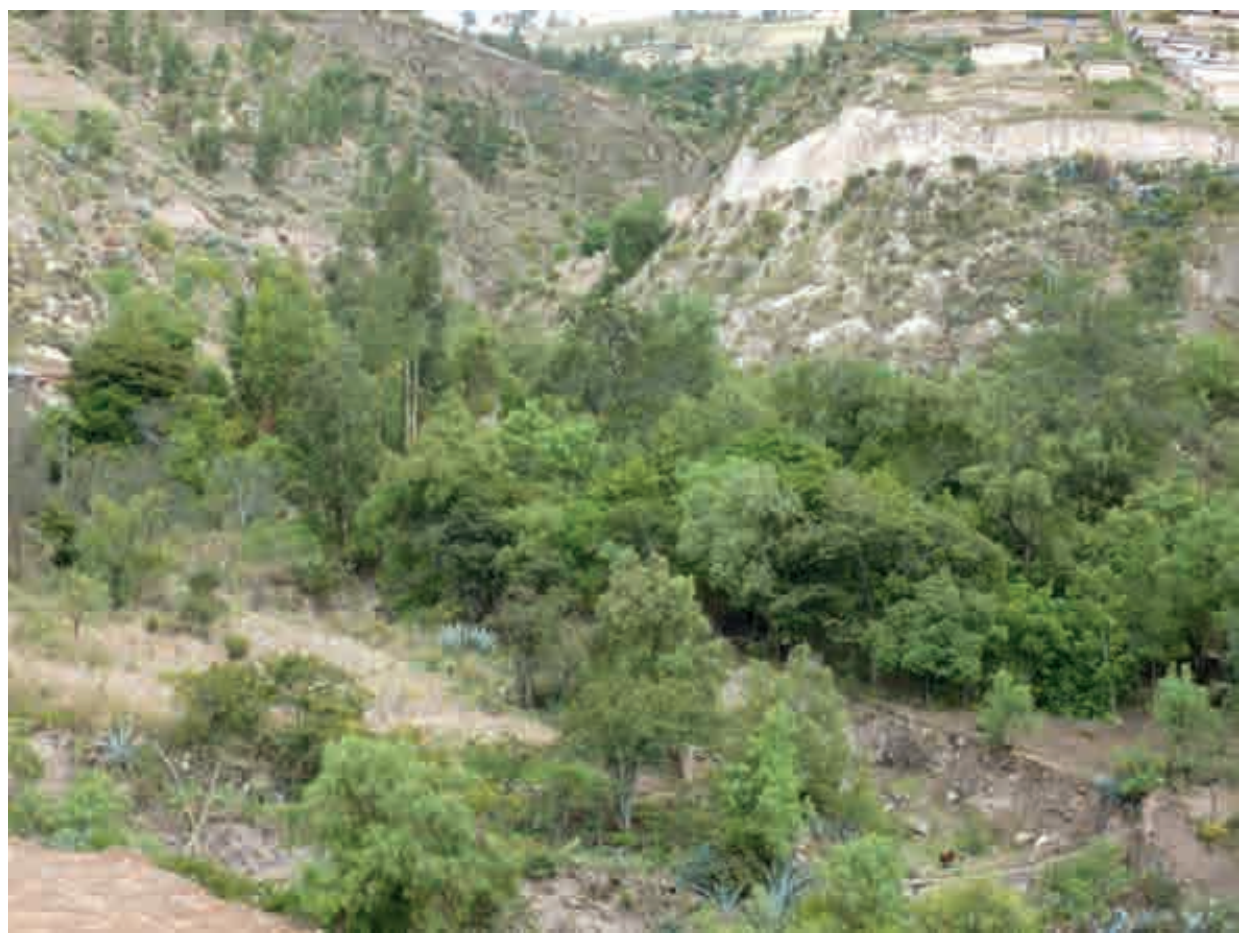

Fig. 4 Zona de Vida: Estepa Espinoso Montano Bajo Subtropical (ee - MBS) en la Q. Pilacucho.

Identificación de tipos de amenazas del Barrio Santa Ana, Pilacucho y Santa Cecilia en la Ciudad de Ayacucho

Los peligros más importantes o de mayor relevancia y recurrencia tenemos los siguientes:

\begin{tabular}{|l|l|}
\hline \multicolumn{1}{|c|}{ TIPO DE AMENAZA Y/O PELIGRO } & \multicolumn{1}{c|}{ DEFINICIÓN } \\
\hline GEOLÓGICAS & $\begin{array}{l}\text { Los movimientos en masa son procesos geoló- } \\
\text { gicos que ocurren por efectos de sismos y que } \\
\text { suelen movilizar masas de rocas y sedimentos } \\
\text { generando inestabilidad-riesgo de derrumbe. }\end{array}$ \\
\hline HIDROMETEOROLÓGICAS & $\begin{array}{l}\text { Las Iluvias intensas estacionales, son irregula- } \\
\text { res que ocasionan la acumulación peligrosa de } \\
\text { agua que acarrean por inundación y huaycos. } \\
\text { Estos eventos de las Iluvias intensas sobre- } \\
\text { pasan la capacidad del suelo por el volumen } \\
\text { máximo de transporte de las quebradas que } \\
\text { desborda e inunda los terrenos y las viviendas. }\end{array}$ \\
\hline
\end{tabular}




\begin{tabular}{|l|l|}
\hline Flujo de lodo y detritus & $\begin{array}{l}\text { Masa de detritos reblandecida por el agua } \\
\text { hasta tener consistencia fluida. Y el flujo de } \\
\text { detritus, son movimientos casi siempre rápi- } \\
\text { dos de rocas y suelo, con predominio de los } \\
\text { materiales gruesos. Pierden su estabilidad es- } \\
\text { tructural y son amenazas para la población. }\end{array}$ \\
\hline Huaycos & $\begin{array}{l}\text { Desplazamiento violento de una gran masa } \\
\text { de Iloclla con lodo y rocas que se movili- } \\
\text { za a gran velocidad por quebradas y va- } \\
\text { Iles. Por Ej. El caso del Alud del Cerro La } \\
\text { Picota de la Ciudad de Ayacucho en 2009 }\end{array}$ \\
\hline SOCIONATURAL & $\begin{array}{l}\text { La población vienen utilizando como mulada- } \\
\text { res de RSU en gran parte toda la quebrada } \\
\text { baja de sector Pilacucho del Barrio Santa Ana, } \\
\text { están expuestas a peligro de contaminación y } \\
\text { salud pública, de tal manera son perjudiciales } \\
\text { para la vida humana, animal, vegetal y otras } \\
\text { obras de infraestructura públicos y privados. }\end{array}$ \\
\hline Efluentes líquidos & $\begin{array}{l}\text { Desagües domiciliarias consistente en aguas } \\
\text { servidas, agua sucia, vertidos tóxicos y todo } \\
\text { tipo derrames domiciliarias que drenan ha- } \\
\text { cia las quebradas y riachuelos, zonas de alta } \\
\text { peligrosidad que contaminan a los lechos. }\end{array}$ \\
\hline
\end{tabular}

\section{CARACTERÍSTICAS DE INFRAESTRUCTURA, SOCIO-DEMOGRÁFICAS DE LA ZONA EN ESTUDIO}

\section{Infraestructura de Servicios Urbanos}

El acceso a la zona es mediante vías pavimentadas y veredas de concreto simple, como también por calles de tierra no asfaltados. Otra de las características de la zona en estudio cuenta con suministro de los servicios básicos de agua, desagüe (70\%), redes de comunicación, energía eléctrica, las viviendas están construidas de material rústico de dos aguas tejado cuyos modelos de vivienda van replicando la vida campesina. La población actual circundante a la zona en evaluación, cuenta con los siguientes datos generales:

Población : : 1,303 habitantes.

Área : 14.05 ha.

$\mathrm{N}^{\circ}$ Lotes : 270 viviendas.

La Altura promedio es de 2,710.690 msnm.

Según estudios de INDECI (2008), tiene una extensión superficial de 14.05 hectáreas representa el 0.76\% de la Geografía Urbana, concentra una población de 1,124 habitantes, que ocupan un promedio de 80 hab/ha. 


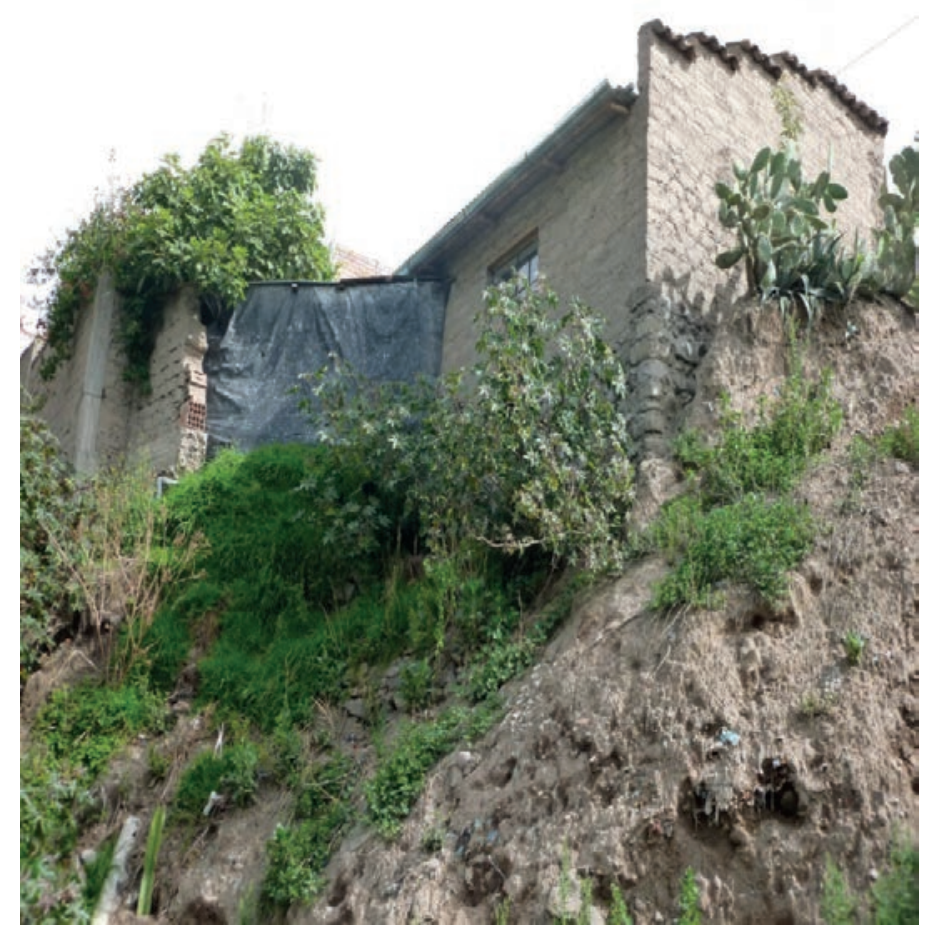

Fig. 8 Casas construidas de material de barro y adobe en los bancos y terrazas deleznables.

\section{DISCUSIÓN}

Las quebradas de "Santa Cecilia" y "Pilacucho", son huaycos secas con cauces regulares bien drenadas, con acumulaciones de sedimentos acarreados y colmatados con grandes cantidades de materiales sedimentarios de distinto granulometría altamente inestable, que en época de lluvias por procesos de escorrentía se van activando y causan inundaciones con descargas fluviales, zonas amenazadas por inundaciones a gran velocidad, con gran fuerza hidrodinámica y poder erosivo y se convierten en un peligro muy alto cuyo valor alcanza de acuerdo al siguiente cuadro:

\section{ESTRATIFICACION}

\begin{tabular}{|c|c|c|c|c|}
\hline PELIGRO & BAJO & MEDIO & ALTO & MUY ALTO \\
\hline & $<25 \%$ & $26-50 \%$ & $51-75 \%$ & $\mathbf{7 6 - 1 0 0 \%}$ \\
\hline VALORACION & & & & $\mathbf{8 5 \%}$ \\
\hline
\end{tabular}

Valoración: 85\%

El Peligro: Inundación = Nivel Muy Alto

El Huayco, son desplazamientos violentos de una gran masa de lloclla con lodo y rocas que se moviliza a gran velocidad por quebradas y valles. Sectores que son inundados a baja velocidad y permanecen bajo el agua por varios días. En los meandros van acumulando gran cantidad de materiales sueltos desde cantos rodados hasta arenas, areniscas y arcillas. 


\section{ESTRATIFICACION:}

\begin{tabular}{|c|c|c|c|c|}
\hline PELIGRO & BAJO & MEDIO & ALTO & MUY ALTO \\
\hline & $<25 \%$ & $26-50 \%$ & $51-75 \%$ & $76-100 \%$ \\
\hline VALORACION & & & $55 \%$ & \\
\hline
\end{tabular}

Valoración: 55\%

El Peligro: Huayco = Nivel Alto

Suelo de calidad intermedia, con aceleraciones sísmicas moderadas, en el sector en análisis se encuentra en una zona de baja a media amplificación sísmica con probabilidad de ocurrencia de Grado VI a VII (MM: Escala Mercalli Modificada) y de medio peligro sísmico.

\section{ESTRATIFICACION:}

\begin{tabular}{|c|c|c|c|c|}
\hline PELIGRO & BAJO & MEDIO & ALTO & MUY ALTO \\
\hline & $<25 \%$ & $26-50 \%$ & $51-75 \%$ & $76-100 \%$ \\
\hline VALORACION & & $35 \%$ & & \\
\hline
\end{tabular}

Valoración: 35\%

El Peligro: Sismo =Nivel Medio

Los residuos urbanos sólidos, es el material o producto cuyo poseedor desecha y que se encuentra en un estado sólido o semisólido, hay desechos orgánicos, inorgánicos y tóxicos que puede ser susceptible de ser valorizado o requiere sujetarse a tratamiento o disposición final, todo el cauce de Pilacucho está contaminado.

ESTRATIFICACION:

\begin{tabular}{|c|c|c|c|c|}
\hline PELIGRO & BAJO & MEDIO & ALTO & MUY ALTO \\
\hline & $<25 \%$ & $26-50 \%$ & $51-75 \%$ & $76-100 \%$ \\
\hline VALORACION & & & $70 \%$ & \\
\hline
\end{tabular}

Valoración: $70 \%$

El Peligro: Contaminación Ambiental = Nivel Alto.

Análisis de vulnerabilidad

La vulnerabilidad, es el grado de debilidad o exposición de un elemento o conjunto de elementos frente a la ocurrencia de un peligro natural o antrópico. La vulnerabilidad de la Quebrada de Pilacucho, presenta aire contaminado y arrojo constante de basuras en todo el cauce, las construcciones de las viviendas es de adobe carentes de servicios de alcantarillas, por su marginalidad están ubicadas en toda la quebrada. La población económicamente es frágil, por ser pequeños productores de artesanías ligados temporalmente al mercado exterior. Políticamente las decisiones políticas y de gestión carecen de coordinación con las autoridades de los Comités de Defensa Civil, socialmente están en situación de pobreza débiles de organización en temas de prevención de desastres y por falta de una cultura de riesgo - desastre. La estimación y análisis de vulnerabilidad se resumen en el siguiente cuadro: 
CUADRO N ${ }^{\circ}$ : RESUMEN GENERAL DE LAS VULNERABILIDADES POR INUNDACION

\begin{tabular}{|c|c|c|c|c|}
\hline \multirow{2}{*}{ VULNERABILIDAD } & \multicolumn{4}{|c|}{ VALORACION } \\
\cline { 2 - 5 } & VB: $\mathbf{< 2 5 \%}$ & $\begin{array}{c}\text { VM: } \mathbf{2 6}- \\
\mathbf{5 0 \%}\end{array}$ & VA: $\mathbf{5 1 - 7 5 \%}$ & VMA: $\mathbf{7 6 - 1 0 0 \%}$ \\
\hline ECOLOGICA Y AMBIENTAL & & & $\mathbf{7 3 . 6 0}$ & \\
\hline FISICA & & & & $\mathbf{8 1 . 7 0}$ \\
\hline ECONÓMICA & & & $\mathbf{7 3 . 5 0}$ & \\
\hline POLITICA E INSTITUCIONAL & & & $\mathbf{6 1 . 3 3}$ & \\
\hline SOCIAL & & & & $\mathbf{8 5 . 0 0}$ \\
\hline EDUCATIVA & & & $\mathbf{7 5 . 0 0}$ & \\
\hline
\end{tabular}

DONDE:

Vulnerabilidad Total: $\mathrm{VT}=0.7 \mathrm{VF}+0.3 \mathrm{Vs}$ (1)

$\mathrm{Vs}=(\mathrm{VEA}+\mathrm{VE}+\mathrm{VPI}+\mathrm{VS}+\mathrm{VED}) / \mathrm{N}$.

$\mathrm{Vs}=(73.60+73.50+61.33+85.00+75.00) / 5=73.70 \%$

Entonces: $\mathrm{VT}=0.7 \times 79.81 .70+0.3 \times 73.70=80.00 \%$

VT $=80.00 \%$ : Vulnerabilidad Muy Alta

La vulnerabilidad del Peligro de INUNDACIÓN: MUY ALTO

CUADRO N²: RESUMEN GENERAL DE LAS VULNERABILIDADES DE CONTAMINACION AMBIENTAL

\begin{tabular}{|c|c|c|c|c|}
\hline \multirow{2}{*}{ VULNERABILIDAD } & \multicolumn{4}{|c|}{ VALORACION } \\
\cline { 2 - 5 } & VB: $\mathbf{< 2 5 \%}$ & $\begin{array}{c}\text { VM: } \mathbf{2 6 -} \\
\mathbf{5 0 \%}\end{array}$ & VA: $\mathbf{5 1 - 7 5 \%}$ & VMA: $\mathbf{7 6 - 1 0 0 \%}$ \\
\hline ECOLOGICA Y AMBIENTAL & & & $\mathbf{7 5 . 0 0}$ & \\
\hline FISICA & & & $\mathbf{7 5 . 0 0}$ & \\
\hline ECONÓMICA & & & $\mathbf{7 3 . 5 0}$ & \\
\hline POLITICA E INSTITUCIONAL & & & $\mathbf{6 1 . 3 3}$ & $\mathbf{8 5 . 0 0}$ \\
\hline SOCIAL & & & & \\
\hline EDUCATIVA & & & $\mathbf{7 5 . 0 0}$ & \\
\hline
\end{tabular}

DONDE:

Vulnerabilidad Total: $\mathrm{VT}=0.7 \mathrm{VEA}+0.3 \mathrm{Vs}$

$\mathrm{Vs}=(\mathrm{VF}+\mathrm{VE}+\mathrm{VPI}+\mathrm{VS}+\mathrm{VED}) / \mathrm{N}=(75.00+73.50+61.33+85.00+75.00) / 5=80.00 \%$

Entonces: $\mathrm{VT}=0.7 \mathrm{x} 75.00+0.3 \times 80.00=77.80 \%$

VT = 77.80\%: Vulnerabilidad Muy Alta

La Vulnerabilidad del Peligro por CONTAMINACIÓN AMBIENTAL: MUY ALTO. 
El riesgo4 con respecto a la INUNDACIÓN, según los análisis efectuados de las vulnerabilidades es de RIESGO MUY ALTO.

Los causes de las quebradas evaluadas, han sido ocupados por viviendas mayormente rústicos de familias de extrema pobreza, que en épocas de lluvias se activan con la caída de huaycos en grandes proporciones que en muchas oportunidades han causado la inundación. En los momentos actuales, el riesgo viene incrementándose por la construcción de nuevas viviendas en el cauce natural de las quebradas habiendo reducido el ancho de los mismos, como también la presencia de desmontes y desechos sólidos han colmatado el cauce natural y algunas viviendas han quedado por debajo del nivel actual.

Para determinar los niveles de riesgo también se puede utilizar la Matriz de doble entrada, conociendo los valores de los peligros y vulnerabilidades.

CUADRO N ${ }^{\circ}$ 3: MATRIZ DE PELIGRO Y VULNERABILIDAD

\begin{tabular}{|c|c|c|c|c|}
\hline Peligro Muy Alto & Riesgo Alto & Riesgo Alto & Riesgo Muy Alto & Riesgo Muy Alto \\
\hline Peligro Alto & Riesgo Medio & Riesgo Medio & Riesgo Alto & Riesgo Muy Alto \\
\hline Peligro Medio & Riesgo Bajo & Riesgo Medio & Riesgo Medio & Riesgo Alto \\
\hline Peligro Bajo & Riesgo Bajo & Riesgo Bajo & Riesgo Medio & Riesgo Alto \\
\hline & $\begin{array}{c}\text { Vulnerabilidad } \\
\text { Baja }\end{array}$ & $\begin{array}{c}\text { Vulnerabilidad } \\
\text { Media }\end{array}$ & $\begin{array}{c}\text { Vulnerabilidad } \\
\text { Alta }\end{array}$ & $\begin{array}{c}\text { Vulnerabilidad } \\
\text { Muy Alta }\end{array}$ \\
\hline
\end{tabular}

LEYENDA: $\square$ Riesgo Bajo (< de 25\%)

Riesgo Medio (26\% al $50 \%$ )

Riesgo Alto (51\% al $75 \%)$

Riesgo Muy Alto (76\% al $100 \%)$

R = P X V --------------- Inundación

$\mathrm{R}=$ Peligro Muy Alto (85\%) x Vulnerabilidad Muy Alto (80.00\%)

Riesgo = Muy Alto

La población potencialmente afectable por el peligro de INUNDACION viene a ser 120 familias y/o 600 habitantes.

$\mathrm{R}=\mathrm{PMA} x \mathrm{VMA}=\mathrm{RMA}=76 \%-100 \%$.

Damnificados $=456$ a 600 habitantes (91 a 120 viviendas).

La pérdida de daños por inundación, considerando el valor promedio de una vivienda $S / .5,000$ se estima en:

4. El riesgo se define como "la probabilidad de daños o pérdidas ocasionados en vidas humanas (muertes, heridos), medios económicos (propiedades, actividad económica) y el ambiente, como resultado de la interacción entre peligros de origen natural, socionatural o antropogénico y condiciones de vulnerabilidad" (GTZ, 2007). 
Pérdidas económicas: 455,000.00 a 600,000.00 nuevos soles.

El riesgo con respecto a la CONTAMINACIÓN AMBIENTAL, según los análisis efectuados de las vulnerabilidades es de ALTO RIESGO.

Cabe señalar en el tema de manejo de residuos sólidos de las personas y/o habitantes circundantes en la zona en estudio, estos arrojan constantemente desechos orgánicos (desechos alimenticios, heces, etc.) e inorgánicos (botellas de vidrios, maderas, latas, prendas viejas, etc.) al cauce de las quebradas; como también hay viviendas nuevas construidas, y muchos de ellos en el mismo cauce de las quebradas, donde su habitantes vierten las aguas residuales hacia las quebradas a través de tuberías de PVC de diferentes diámetros, las mismas que sirven de medios de cultivo de microorganismos propagados por los agentes vectores de enfermedades, como: insectos, moscas, roedores, canes, etc., situación que trae como consecuencia la propagación de enfermedades diarreicas agudas (EDAS), infecciones respiratorias agudas (IRAS), enfermedades dérmicas, entre otros.

Si de una población total de 14,192 habitantes, acudieron a los Centros de Salud de Belén y Santa Ana con enfermedades diarreicas agudas (EDA), infección respiratoria aguda (IRA), etc. una cantidad de 5,937 personas, entonces específicamente la población en el ámbito del estudio referente al tema de contaminación ambiental es de 1,303 personas, que representa 261 familias y el cálculo se realiza en base a esta cantidad.

Luego de realizado el análisis de la zona en riesgo se tiene que el $\mathrm{R}=$ Alto, Esto quiere decir que: $\mathrm{R}=76$ al 100\%. Si se tiene que en la zona hay 261 viviendas. ¿Cuántos de ellos serían los afectados?

Para ello se aplica el rango del 76 al 100\%. Quiere decir que el número de viviendas que podrían ser afectados serían:

El $76 \%$ de $261=198$

El $100 \%$ de $261=261$

Si consideramos a 05 habitantes por viviendas, cuántos serían los afectados?

$198 \times 5=990$

$261 \times 5=1,303$.

Las consecuencias de la contaminación ambiental trae enfermedades como IRA, EDA, y por decir consideramos para su tratamiento el costo mínimo de S/.220.00 por persona. Entonces estamos hablando de un riesgo en la salud que oscila entre gasto de s/. 217,800.00 a s/ 286,660.00 que vendría a ser el costo por Contaminación Ambiental.

Para determinar los niveles de riesgo también se puede utilizar la Matriz de doble entrada, conociendo para los valores de los peligros y vulnerabilidades. 
CUADRO N 4: MATRIZ DE PELIGRO Y VULNERABILIDAD

\begin{tabular}{|c|c|c|c|c|}
\hline Peligro Muy Alto & Riesgo Alto & Riesgo Alto & Riesgo Muy Alto & Riesgo Muy Alto \\
\hline Peligro Alto & Riesgo Medio & Riesgo Medio & Riesgo Alto & Riesgo Muy Alto \\
\hline Peligro Medio & Riesgo Bajo & Riesgo Medio & Riesgo Medio & Riesgo Alto \\
\hline Peligro Bajo & Riesgo Bajo & Riesgo Bajo & Riesgo Medio & Riesgo Alto \\
\hline & $\begin{array}{c}\text { Vulnerabilidad } \\
\text { Baja }\end{array}$ & $\begin{array}{c}\text { Vulnerabilidad } \\
\text { Media }\end{array}$ & $\begin{array}{c}\text { Vulnerabilidad } \\
\text { Alta }\end{array}$ & $\begin{array}{c}\text { Vulnerabilidad } \\
\text { Muy Alta }\end{array}$ \\
\hline
\end{tabular}

LEYENDA: $\square$ Riesgo Bajo (< de 25\%)

$\square$ Riesgo Medio (26\% al 50\%)

Riesgo Alto (51\% al $75 \%)$

$\square$ Riesgo Muy Alto (76\% al $100 \%)$

R = P x V ---------------- Contaminación Ambiental

$\mathrm{R}=$ Peligro Alto (70\%) x Vulnerabilidad Muy Alto (77.80)

Riesgo $=$ Alto

Como conclusiones permite determinar el grado de afectación y pérdida, que podría resultar de la ocurrencia de un evento adverso en la ciudad:

En base al análisis de riesgo, la Quebrada de Pilacucho resulta ser una zona crítica expuestos a dos peligros recurrentes de mayor incidencia como la inundación y la contaminación ambiental, representan riesgos muy altos para la población, procesos que se ve favorecido por una alta pendiente del terreno, baja o pobre resistencia de los materiales que significa baja capacidad portante del suelo deleznable y del agua de lluvia en su acción de erosión, transporte y deposición que inundan los cauces de la quebradas vulnerando las viviendas y todo tipo de infraestructura física los cuales carecen de un adecuado control de planeamiento urbanístico y la persistencia de inadecuado manejo de los residuos sólidos, van contaminando con el vertimiento de aguas residuales a las Quebradas de Santa Cecilia, Pilacucho y Santa Ana.

Las amenazas cotidianas se asocian con la pobreza y la vulnerabilidad en los asentamientos humanos. Entre las amenazas cotidianas más frecuentes son saneamiento y alcantarillado inadecuado, aire contaminado y residuos sólidos urbanos, las viviendas son colapsables, inseguridad de la infraestructura por el tipo de construcción y materiales de adobe. El grado de índice de vulnerabilidad, obedece a las características económicas de familias pobres, socialmente tienen escasa organización social y no coordinan con las autoridades sobre la prevención y atención de desastres; en consecuencia, el índice de vulnerabilidad es de $80.00 \%$, considerada vulnerabilidad muy alta, que significa poblaciones con deficientes respuesta para enfrentar a los efectos negativos de los desastres naturales y ambientales.

\section{AgRADECIMIENTO}

A la población del Barrio de Santa Ana por su colaboración en el trabajo de campo y la recopilación de la información, también de manera particular al Ing. César Inga Oriundo que ha contribuido con 
los planos de peligros y vulnerabilidades. Expresamos nuestro agradecimiento a la Oficina General de Investigaciones e innovación de la $\mathrm{UNSCH}$.

\section{REFERENCIA BiBLIOGRÁFICA}

INGEMET. FOTOGRAFÍA AEREA.

Servicio Aerofotográfico Nacional, Código 181-70 1584, Fecha de toma 17-09-1970. INGEMET, Lima. LEÓN NINA, F.

2009 La Erosión Antrópica y Degradación del Ecosistema en el AA. HH. Cerro La Picota,Ayacucho. Ayacucho: OGII.

LÓPEZ JUÁRES, A.

2013 Manual Para La Evaluación de Riesgos Originados por Fenómenos Naturales. Lima: Editado en CENEPRED.

MARTÍNEZ RUBIANO, M.

2009 Los géografos y la teoría de riesgos y desastres ambientales. Perspectiva Geográfica, 248256.

MATOS ESPINOZA, E.

2003 Mapa de Peligros de la Ciudad Ayacucho. Lima: Proyecto INDECI- PNUD PERÚ.

NARVÁEZ, L.

2009 La gestión del riesgo de desastre: un enfoque basado en proceso. Lima: Editorial PULL CRLATVOS S.A.L.

OCHARAN, J.

2008 Guía Práctica de Reducción del Riesgo de Desastres para Organizaciones Humanitarias y del Desarrollo. Barcelona: Edición Fundación “la Caixa”.

PREDES.

2010 Centro de Estudios y Prevención de Desastres. Lima: PREDES. 